\title{
Parental Acceptance, Mental Health and Self-efficacy of Adults in Bangladesh
}

\author{
Rumana Aktar ${ }^{1}$, Aynur Nahar ${ }^{2}$ \\ ${ }^{1,2}$ Department of Psychology, Faculty of Biological Sciences, University of Chittagong, Chittagong-4331, \\ Bangladesh
}

\begin{abstract}
The present study explored the relation of remembered parental (paternal and maternal) acceptance in childhood with mental health and self-efficacy of adults in Bangladesh. The sample consisted of 150 adults (50\% women) ages 18 through 25 years $(M=22.01$ years) from Bangladesh. The measures used were the adapted Bangla version of Adult version (standard form) of the Parental Acceptance-Rejection Questionnaire (Adult PARQ: Mother \& Father), adapted Bangla version of the Self-efficacy Scale, adapted Bangla version of General Mental Health Questionnaire (GHQ-12). Results of the correlation analyses indicated that remembered maternal and paternal acceptances were significantly correlated with adults' mental health and self-efficacy. Results of multiple regression analyses revealed that maternal and paternal acceptance were significant predictors of mental health and self-efficacy. The present findings may be useful to researchers and practitioners who are concerned with impacts of childhood attachment relationships on the psychological wellbeing and quality of life of people.
\end{abstract}

Keywords: Adults, Maternal acceptance, Mental health, Paternal acceptance and Self-efficacy

\section{Introduction}

The foundation of development of individual lies in the womb of the family. In family specially parents serves as an effective agent of socialization. The form of communication between a parent and a child has a reciprocal effect [1]. Children everywhere need a specific form of positive response i.e. acceptance from parents and other attachment figures. In adulthood, the need becomes more complex and differentiated to include the wish (recognized or unrecognized) for positive regard from people with whom one has an affectional bond of attachment. When this need is not met satisfactory, children worldwide-regardless of variations in culture, gender, age, ethnicity or other such defining conditions tend to report themselves to be hostile and aggressive, dependent or defensively independent, over impaired in self- adequacy and self-esteem and to have a negative worldview among other responses.

Rohner [2], [3], [4] formulated the Parental Acceptance-Rejection Theory (PAR Theory) which is robust and used worldwide regardless of differences in culture, race, gender, geographic context or such defining conditions. The Parental acceptance-rejection theory (PAR Theory) is an evidence-based theory of socialization and lifespan development that seeks to predict and explain major causes, consequences, and other correlates of parental acceptance and rejection worldwide. The theory predicts that parental acceptance-rejection has consistent effects on personality dispositions, psychological adjustment and behavioral functioning of both children and adults worldwide [5].

In PARQ Theory, together parental acceptance and rejection form the warmth dimension of parenting with parental acceptance at the positive end of the continuum and parental rejection at the negative end. The warmth dimension has to do with the quality of the affectional bond between parents and their children, and with the physical, verbal and symbolic behaviors parents use to express these feelings. Parental acceptance refers to the love, affection, care, comfort, support, nurturance and other such behaviors that parents can feel or express toward their children. On the other hand parental rejection refers to the absence of withdrawal of warmth, love, support or affection by parents toward their children. Parental rejection can be shown anywhere in the world by any combination of four principal expressions: (1) cold and unaffectionate, the opposite of being warm and affectionate, (2) hostile and aggressive, (3) indifferent and neglecting, and (4) undifferentiated rejecting. Undifferentiated rejection refers to individuals' beliefs that their parents do not really care about them or love them, even though there might not be clear behavioral indicators that the parents are neglecting, unaffectionate, or aggressive toward them. In PAR theory there are three sub-theories. These include personality sub-theory, coping sub-theory, and socio-cultural systems sub-theory.

Mental health is a state of well-being in which the individual realizes his or her own abilities, can cope with the normal stresses of life, can work productively and fruitfully, and is able to make a contribution to his or her community [6]. Mental Health includes a number of dimensions: self esteem, realization of one's potential, the ability to maintain fulfilling meaningful relationship and psychological well being [7]. It is clear that such a 
view of mental health constitutes for more than the mere absence of illness or disorder. Most people would probably fall for short of optimum mental health including that mental health is not a statistical norm but a goal toward which strive. Consequently, advocates of mental health focus on a broad delivery of mental health services to the general population not just to the most seriously disturbed people. They also emphasize prevention, education as well as intervention that aid people who have already developed particular type of mental illness. There are different types of mental health problems, some of which are common, such as depression and anxiety disorders, and some not so common, such as schizophrenia and Bipolar disorder.

Self-efficacy are salient factors in child development. Self-efficacy also called perceived ability, refers to the confidence people have in their abilities for success in a given task [8]. Individuals who possess a high degree of self-efficacy are more likely to attempt challenging tasks and to exert more effort in the process. If highly efficacious individuals fail, they attribute the outcome to a lack of effort or an adverse environment. When they succeed, they credit their achievement to their abilities. It is the perception that their abilities caused the achievement that affects the outcome rather than their actual abilities [8]. Although inefficacious individuals usually avoid challenging tasks, when they do attempt them they give up more easily than individuals with high efficacy. When inefficacious individuals fail, they attribute the unsuccessful result to a lack of ability and tend to lose faith in their capabilities. When they succeed, they are more likely to attribute their success to external factors [8], [9]. Four factors determine self-efficacy: enactive mastery experience, vicarious experience, verbal persuasion, and physiological and emotional states [8], [9]. Self-efficacy affects every area of human endeavor, by determining the beliefs a person holds regarding his or her power to affect situations.

Several numbers of studies explored the relation of parental acceptance- rejection, mental health and self-efficacy in various cultures. A meta-analysis of 43 studies drawn from 7,563 respondents in 15countries [5] confirmed the expectation that perceived parental acceptance is universally associated with psychological adjustment (or mental health) of both children and adults. Furthermore, strong evidence supports PAR Theory's expectations that children everywhere who come from loving (accepting) families are more likely than children who come from unloving (rejecting) families to feel good about themselves, feel competent, have less problems with the management of hostility and aggression, have adequate emotional responsiveness and emotional stability, have less dependence and have a positive worldview [10], [11], [12]. According to Rohner and Britner their Cross-cultural and intra-cultural evidence converges on the conclusion shows that four classes of mental health issues (psychological adjustment, unipolar depression and depression effect, behavior problems and substance abuse) are possible worldwide correlates of parental acceptance-rejection. Their research also accumulates strongest evidence supporting parental acceptance-rejection theory's personality sub-theory that postulates a universal relationship between perceived parental acceptance-rejection and psychological adjustment. Substantial evidence also supports the likelihood of worldwide correlations between parental acceptance-rejection and three other mental health issues [13]. A cross-cultural research showed that parental acceptance-rejection differs across countries. Parental rejection was associated with adolescent psychological disorders and parental acceptance was associated, to a lesser extent, with better psychological adjustment [14]. Negative parenting and rejection are major variables that influence the development of children and their mental and psychological health during adolescence and adulthood and those adults who perceived their fathers as providing rejection in their childhood, their psychological adjustment was poorer than those who were given acceptance [15]. In a study the effects of perceived maternal and paternal acceptance, power, and prestige was investigated on children's psychological adjustment with a sample of 254 children in Bangladesh [16]. The findings indicated that both maternal and paternal acceptance make independent contributions to children's psychological adjustment (a constellation of seven specific personality dispositions i.e. hostility/aggression, dependency, feelings of impaired self-esteem, feelings of impaired self-adequacy, emotional unresponsiveness, emotional instability and negative worldview).

The relationship between parental acceptance-rejection and self-efficacy of adolescents was investigated on Pakistani samples in several studies. The results of these studies indicated that there is a significant positive relationship between mother and fathers' warmth and general self-efficacy of adolescents [17], [18] as well as there is a significant negative relationship between parent's rejections and self-efficacy of adolescents [18]. These research findings highlight significance of parental acceptance rejection in adolescents' psychological development. It appears from the above literature that there is a relation between parental acceptance, mental health and self-efficacy. Therefore no research has so far been carried out to directly address this issue in the cultural context of Bangladesh. Now the research question is that, is the mental health and selfefficacy of adults significantly affected by the remembered parental acceptance within the family of Bangladesh?

\subsection{Rationale of the Study}

Healthy and warm relation of parents with their children can have profound effects on personality as well as psychological adjustment of their children. The results of present research would be beneficial for 
parents as if it would provide knowledge to them about the importance of parental attitude to the mental development of their offspring. This study will help to generate research findings regarding parental acceptance, mental health development of adult in the context of Bangladesh. Additionally, it would be useful for mental health professionals to consider the contribution of parenting while dealing with their clients.

\subsection{Objectives}

The specific objectives of the study were to explore-

$>$ The relation between remembered parental (maternal and paternal) acceptance and mental health of adults.

$>$ The relation between remembered parental (maternal and paternal) acceptance and self-efficacy of adults.

\subsection{Participants}

\section{Method}

The sample consisted of 150 adult students of University of Chittagong in Bangladesh. Their age ranged from 18 to 25 years with a mean of 22.01 years $(S D=1.52)$. Educational level varied from $1^{\text {st }}$ year (of Honors) to Masters. They were selected conveniently from several departments of University of Chittagong in Bangladesh. Only respondents who grew up in intact nuclear families or larger family unit with their mothers and father in residence during childhood were included in this study. All participants were the biological offspring of their resident parents. About $85 \%$ of the participants were from middle class family background. Other details of the sample characteristics are shown in Table 1 below.

Table 1: Distribution of Respondents by Gender, Parental education and Occupation

\begin{tabular}{llcc}
\hline \multicolumn{1}{c}{ Variables } & & $n$ & $\%$ \\
\hline Respondent's Gender & Men & & 50 \\
& Women & 75 & 50 \\
Maternal Education & Below Secondary (I-X) & 75 & 22.7 \\
& Secondary (IX-X) & 34 & 37.3 \\
& Higher Secondary (XI-XII) & 56 & 12.0 \\
& Bachelor & 40 & 1.3 \\
Paternal Education & Masters & 18 & 8.7 \\
& Below Secondary (I-X) & 2 & 12.0 \\
& Secondary (IX-X) & 13 & 24.7 \\
& Higher Secondary (XI-XII) & 18 & 42.0 \\
& Bachelor & 37 & 12.0 \\
Maternal Occupation & Masters & 63 & .7 \\
& Above Masters & 18 & 88.0 \\
Paternal Occupation & Homemaking & 1 & 12.0 \\
& Service & 132 & 38.7 \\
& Business & 18 & 54.0 \\
\hline
\end{tabular}

\subsection{Measures}

All the respondents in this study responded to the following four self report questionnaire along with personal information form. (1) Adult version of the Parental Acceptance-Rejection Questionnaire for Fathers (Adult PARQ: Father [19], (2) Adult version of the Parental Acceptance-Rejection Questionnaire for Mothers (Adult PARQ: Mother [19], (3) Global Mental Health Questionnaire (GHQ-12), [20], (4) General Self-efficacy Scale [21]. All questionnaires were translated and adapted into Bangla language and culture.

\subsubsection{Adult version (standard form) of the Parental Acceptance-Rejection Questionnaire (Adult PARQ: Mother \& Father [19])}

The Bangla version [22] of PARQ: Mother and Father [19] were used. These self-report measures consist of 60 items each. The two versions are virtually identical except for reference to mother's behavior versus father's behavior and are designed to measure individuals' perceptions of maternal and paternal acceptance or rejection. In both, respondents are asked to reflect on their perceptions of their mothers' or fathers' behaviors toward them when they were about 7 through 12 years old. This means respondents should answer in the way they really feel, not the way might (have) like (d) their parents to be, or the way might now like to be as a parent. Both measures consist of four subscales each: (a) paternal or maternal Warmth/Affection (20 items), (b) Hostility/Aggression (15 items), (c) Indifference/Neglect (15 items) and (d) Undifferentiated 
Rejection (10 items). In both versions items are scored on 4-point Likert-type scales with 4 (almost always true), 3 (sometimes true), 2 (rarely true) and 1 (almost never true). Scores on the four acceptance-rejection scales are summed after reverse scoring the Warmth/Affection scale to create a measure of coldness and lack of affection. Similarly seven of the items on the indifference/neglect scale (which are worded positively) must be reverse scored to make them consistent with the other negatively worded items on that scale. This produces an overall measure of perceived acceptance-rejection that ranges from a low of 60 (maximum perceived acceptance) to a high of 240 (maximum perceived rejection). The ideal form of the Adult PARQ is designed conceptually in such a way that scores at or above 150 reveals the experience of significantly more rejection than acceptance. Scores between 140 and 149 indicates that the respondents experience high level rejection, but not more overall rejection than acceptance. Scores between 121 and 139 indicate moderate rejection. On the other hand, scores between 60 and 120 reveal individuals' perception of parental love. The Coefficient alphas in this study were .92 for the mother version and .93 for the father version.

\subsubsection{Global Mental Health Questionnaire (GHQ-12) [20]}

Bengali version of general mental questionnaire (GHQ-12) item version [23] originally developed by [20] was used to measure mental health of participants. It is concerned with two major phenomena. The inability to carry out one's normal healthy functions and the appearance of new phenomena of distressing nature. The scale contains 6 positive and 6 negative items. The items score on 4-point Likert- types scale ('not at all', 'somewhat', 'to a considerable extent' and 'to a great extent'). Positive items were scored from 0 to 3 . For negative items, scoring was in reverse order. The sum of scores of all items was the total score of the scale with a range of 0 to 36 for an individual. Higher scores indicate better mental health. Coefficient alpha for the questionnaire was .86 in this study.

\subsubsection{General Self-efficacy Scale [21]}

Bangla version of the General self-efficacy scale [24], originally developed by [21] was used to measure general level of belief in one's competence. It is a 17 items 5 point- Likert type scale. The items were answered on a five-point response format with 1 (disagree strongly), 2 (disagree moderately), 3 (neither agree nor disagree), 4 (agree moderately), 5 (agree strongly). The scale contains 7 positive \& 10 negative items. For negative items, scoring was in reverse order. The sum of scores of all items was the total score of the scale of an individual. Higher scores indicate higher self-efficacy. Coefficient alpha for the questionnaire in the study was .74 .

\subsubsection{Personal Information Form}

This questionnaire elicited demographic, personal and social information that included respondents' age, gender, birth order, number of siblings, father`s \& mother`s educational qualification \& occupation, family income, family member, religion.

\subsection{Design of the Study}

Cross sectional survey design was used in this study.

\subsection{Procedure}

Data were collected individually from each respondent. The respondents were informed about the purpose of the study and necessary rapport was established with respondents. Questionnaires were given to these who were agreed and were interested to participate in the study. The respondents were instructed to read the items of the scales attentively and to respond rapidly. They were also requested not to omit any item in the questionnaire and were told that there was no right or wrong answer. They were assured that the data would be kept confidential and would be used only for research purpose. The respondents did not show any resentment. To complete the questionnaire, most respondents took half an hour. After the completion, every respondent were thanked for their cooperation and participation in the study.

\section{Results}

Adults' mean score for remembered maternal acceptance $(M=106.97, S D=22.35, n=150)$ indicates that adults in this sample experienced, on average, more maternal acceptance than rejection in childhood. Eight respondents $(5.33 \%)$ of the sample had PARQ scores (mother version) at or above 150, indicating that they experienced more maternal rejection than acceptance. Adults' mean score for remembered paternal acceptance $(\mathrm{M}=108.92, S D=24.77, n=150)$ indicates that adults in this sample experienced on the average more paternal acceptance than rejection in childhood. Eight respondents $(5.33 \%)$ of the sample had PARQ scores (father version) at or above 150, indicating that they experienced more paternal rejection than acceptance. 
We computed descriptive statistics, e.g. means and standard deviations for remembered maternal and paternal acceptance, mental health, and self-efficacy both for men and women. We also computed independent samples $t$ values to test gender differences among variables. The results are presented in Table 2 .

Table 2: Descriptive Statistics and Gender Differences in Measures of Perceived Maternal and Paternal Acceptance, Mental Health \& Self-efficacy

\begin{tabular}{|c|c|c|c|c|}
\hline Measures & $M$ & $S D$ & $n$ & $t$ \\
\hline \multicolumn{5}{|l|}{ Maternal acceptance } \\
\hline Women & 109.68 & 25.08 & 75 & \multirow[b]{2}{*}{1.493} \\
\hline Men & 104.25 & 19.02 & 75 & \\
\hline \multicolumn{5}{|l|}{ Paternal acceptance } \\
\hline Women & 108.92 & 24.97 & 75 & \multirow{2}{*}{.000} \\
\hline Men & 108.92 & 24.74 & 75 & \\
\hline \multicolumn{5}{|l|}{ Global Mental Health } \\
\hline Women & 22.21 & 7.26 & 75 & \multirow[b]{2}{*}{$-1.97 *$} \\
\hline Men & 24.27 & 5.48 & 75 & \\
\hline Women & 52.65 & 9.75 & 75 & \multirow[b]{2}{*}{$-3.30 * *$} \\
\hline Men & 56.89 & 7.16 & 75 & \\
\hline
\end{tabular}

$* p=.05, * * p=.003, d f=148$

Table 2 shows that, both men and women perceived their mothers as well as fathers to be fairly accepted. No significant gender difference was found in perceived maternal and paternal acceptance. It is evident from Table 2 that there was significant gender difference in mental health and self-efficacy. Men $(M=24.27, S D=5.48)$ show better mental health than women $(M=22.21, S D=7.26)$. Women $(M=52.65$, $S D=9.75)$ have lower self-efficacy compared to men $(M=56.77, S D=7.16)$.

Thus there were no significant gender differences in maternal and paternal acceptance, so correlations were computed for whole sample to determine the relationship among the variables showed in Table 3.

Table 3: Correlations between Maternal and Paternal Acceptance, Global Mental Health and Self-efficacy

\begin{tabular}{|c|c|c|c|c|}
\hline Variables & 1 & 2 & 3 & 4 \\
\hline 1.Maternal Acceptance & --- & & & \\
\hline 2.Paternal Acceptance & $.461 * *$ & --- & & \\
\hline 3.Mental Health & $-.510 * *$ & $-.470 * *$ & --- & \\
\hline 4.Self-efficacy & $-.434 * *$ & $-.454 * *$ & $.589 * *$ & --- \\
\hline
\end{tabular}

$* * p=.01$

Table 3 reveals that both maternal acceptance and paternal acceptance (lower scores indicate more parental acceptance) were significantly negatively correlated with mental health (higher scores indicate better mental health). It also shows that self-efficacy (higher scores indicate higher self-efficacy) significantly negatively correlated with both maternal acceptance and paternal acceptance (lower scores indicate more parental acceptance).

The results of regression analyses of mental health and self-efficacy on parental acceptance are presented in Table 4 and Table 5 respectively.

Table 4: Multiple Regression Analyses (enter method) of Mental Health on Parental Acceptance (Maternal and Paternal Acceptance)

\begin{tabular}{lccccc}
\hline \multicolumn{1}{c}{ Variables } & $B$ & $S E B$ & $\beta$ & $t$ & $s r^{2}$ \\
\hline Maternal Acceptance & -.108 & .22 & -.372 & $-4.893^{*}$ & .11 \\
Paternal Acceptance & -.78 & .20 & -.298 & $-3.920^{*}$ & .07 \\
\hline
\end{tabular}

Note. $\quad R^{2}=.330$, Adjusted $R^{2}=.321(F 2,147=36.18, p<.001)$

$s r^{2}=$ Squared semi partial correlation

$* p<.001$

Table 4 indicates that both maternal acceptance $(\beta=-.372, t=-4.893, p<.001)$ and paternal acceptance $(\beta=-.298, t=-3.92, p<.001)$ were the significant predictors of mental health. Adjusted $\mathrm{R}^{2}$ value indicates that maternal \& paternal acceptance jointly explained $32.1 \%$ variance in mental health. Semi partial 
correlation coefficients further more indicate that the unique contribution to the explanation of variance in mental health was $11 \%$ for mother and $7 \%$ for father.

Table 5: Multiple Regression Analyses (enter method) of Self-efficacy on Parental Acceptance (Maternal and Paternal Acceptance)

\begin{tabular}{lccccc}
\hline \multicolumn{1}{c}{ Variables } & $B$ & $S E B$ & $\beta$ & $t$ & \\
\hline Maternal Acceptance & -.112 & .031 & -.285 & $-3.59 *$ & -06 \\
Paternal Acceptance & -.115 & .028 & -.323 & $-4.07 *$ & .08 \\
\hline
\end{tabular}

Note. $R^{2}=.270$, Adjusted $R^{2}=.260(F 2,147=27.21, p<.001)$

$s r^{2}=$ Squared semi partial correlation

$* p<.001$

Table 5 shows that both maternal acceptance $(\beta=-.285, t=-3.59, p<.001)$ and paternal acceptance $(\beta$ $=-.323, t=-4.07, p<.001)$ were the significant predictors of self-efficacy. Adjusted $\mathrm{R}^{2}$ value indicates that maternal \& paternal acceptance jointly explained $26.0 \%$ variance in self-efficacy. Semi partial correlation coefficients further more indicate that the unique contribution to the explanation of variance in self-efficacy was $6 \%$ for mother and $8 \%$ for father.

\section{Discussion}

It was found from the results of the study that both men and women perceived their mothers as well as fathers to be fairly accepted in their childhood which is consistent with some previous research findings on Bangladeshi samples [25], [26]. Moreover there was no significant gender difference between remembered maternal and paternal acceptance, although significant gender differences were found in mental health and selfefficacy. In both cases, men had better mental health and higher general self-efficacy than women. Some existing literature on mental health and self-efficacy exhibited that mental disorder i.e. depression, anxiety, somatoform disorder is more prevalent in women than in men [27] and women have lower self-efficacy in highly esteem and highly paying career and technical performance [28]. The results of correlation analyses of this study revealed that both remembered maternal and paternal acceptance were significantly correlated with mental health and self-efficacy. Furthermore multiple regression analyses indicated that both remembered maternal and paternal acceptance were significant predictors of adults' mental health and self-efficacy. It is to be noted here that contribution of remembered maternal acceptance to mental health was greater than that of remembered paternal acceptance in this study. Additionally, the contribution of remembered paternal acceptance was greater than maternal acceptance to self-efficacy.

It is appeared from the results of the present study that both maternal acceptance and paternal acceptance have significant effect on the mental health which are fairly consistent with several other studies (for example [13], [4]). In addition, the findings indicated that remembered maternal acceptance is a stronger predictor of mental health than remembered paternal acceptance of adults which is not consistent with the previous research finding on Bangladeshi samples, where contribution of father was reported greater [25]. This inconsistency might be attributed to a relatively small sample size of the present study. The sample size of the previous study was 754 and adults' psychological adjustment (one of the four mental health issues according to [13]) was measured in that study.

Results concerning remembered parental acceptance and self-efficacy revealed that both the remembered maternal and paternal acceptance was significant predictor of adults' self-efficacy. These findings are in agreement with the findings of a study conducted on Pakistani samples [29]. Furthermore it was found from the result of the present study that remembered paternal acceptance was a stronger predictor of selfefficacy than maternal acceptance which are in line of some existing literature [30], [31].

Results of the present study are in line with PAR Theory's personality sub-theory which attempts to predict and explain major personality or psychological-especially mental health-related consequences of perceived interpersonal-especially parental acceptance and rejection [4].

\section{Conclusion}

The present study extended the literature by showing the relation of remembered parental acceptance with mental health and self-efficacy of adults in the context of Bangladeshi culture. The present findings may be useful to researchers and practitioners who are concerned with impacts of childhood attachment relationships on the overall psychological well-being in adulthood. However, the present study has several limitations that restrict the generalizability of the findings. First, this is a cross sectional study and not a longitudinal study. So we don't know how the early attachment relationship and its impacts change over the lifespan of an individual. Second, the data are based only on subjective questionnaires. Third, information about early attachment relationships is based only on reminiscence of childhood experiences. Fourth, sample of adults were from a 
particular institution and socioeconomic class not from its full spectrum. Further study is required in Bangladesh in order to conclusively explain the inconsistency to the contribution of remembered maternal acceptance to mental health of adults.

\section{Acknowledgement}

Authors are grateful to all the respondents for their cooperation and participation in this research.

\section{References}

[1] Russo, J. B., \& Owens, R. E. (1982). The development of an observation tool for parent-child interaction. Journal of Speech and Hearing Disorders, 47, 165-173.

[2] Rohner, R. P., \& Khaleque, A. (2006). Handbook for the study of parental acceptance and rejection. Storrs: Rohner Research Publications.

[3] Rohner, R. P., Khaleque, A., \& Cournoyer, D.E. (2007). Introduction to parental acceptance- rejection theory, methods, evidence, and implications. Retrieved May 8, 2007 from the University of Connecticut Center for the Study of Interpersonal Acceptance and Rejection website at www.csiar.uconn.edu.

[4] Rohner, R. P., Khaleque, A., \& Cournoyer, D.E. (2012). Introduction to parental acceptance-rejection theory, methods, evidence, and implications. Retrieved November 16, 2012 from the University of Connecticut Center for the Study of Interpersonal Acceptance and Rejection website at www.csiar.uconn.edu.

[5] Khaleque, A., \& Rohner, R. P. (2002). Perceived parental acceptance-rejection and psychological Adjustment: A meta-analysis of cross-cultural and intercultural studies. Journal of Marriage and Family, 4, 54-64.

[6] World Health Organization, The World Health Report on Mental Health: New Understanding, New Hope (2001). Retrieved from http://www.who.int/whr/2001/en/whr01_ch1_en.pdf

[7] Ryff, C. D. (1995). Psychological well-being in adult life. Current Directions in Psychological Science. 4, 99-104.

[8] Bandura, A. (1997). Self-efficacy: The exercise of control. New York: Freeman.

[9] Bandura, A. (1986). Social foundations of thought and action: A social cognitive theory. Englewood Cliffs, NJ: Prentice-Hall.

[10] Kim, D., \& Rohner, R. P. (2002). Parental warmth, control, and involvement in schooling predicting academic achievement among Korean American adolescents. Journal of Cross-Cultural Psychology, 33, 127-140.

[11] Kim, S., \& Rohner, R. P. (2003). Perceived parental acceptance and emotional empathy among university students in Korea. Journal of Cross-Cultural Psychology, 34, 723-735.

[12] Rohner, R. P. (2004). The "parental acceptance-rejection syndrome": Universal correlates of perceived rejection. American Psychologist, 59, 830-840.

[13] Rohner, R. P., \& Britner, P. A. (2002). Worldwide mental health correlates of parental acceptance-rejection: Review of cross-cultural and intracultural evidence. Cross-Cultural Research, 36, 16-47. doi: 10.1177/106939710203600102

[14] Dwairy, M. (2010). Parental Acceptance-Rejection: A Fourth Cross-Cultural Research on Parenting and Psychological Adjustment of Children, Journal of Child and Family Studies, 19(1), 30-35. doi: 10.1007/s10826-009-9338-y

[15] Yoshizumi, T., Murase, S., Murakami, T., \& Takai, J. (2007). Dissociation as a mediator between perceived parental rearing style and depression in an adult community population using college students. Personality and Individual Differences, 43, 353-364.

[16] Uddin, M. K., Khaleque, A., Aktar, R., \& Hossain. K. N. (in press). Relation between perceived parental acceptance and children's psychological adjustment in the context of differential parental power and prestige. Cross-Cultural Research.

[17] Kazmi, S.R. (2009). Relationship between Parental Acceptance-Rejection and Self efficacy in Adolescents. Unpublished master's thesis, Psychological Abstracts 2003-2009. Department of Applied Psychology, University of the Punjab, Lahore-Pakistan. Retrieved from http://pu.edu.pk/images/Psychological\%20Abstracts\%202003-2009.pdf

[18] Kausar, R., \& Kazmi, S.R. (2011). Perceived Parental Acceptance-Rejection and Self-efficacy of Pakistani Adolescents. Journal of the Indian Academy of Applied Psychology, 37(2), 224- 232.

[19] Rohner, R. P. (2005). Parental acceptance-rejection questionnaire (PARQ): Test manual. In R. P. Rohner, \& A. Khaleque (Eds.). Handbook for the study of parental acceptance and rejection (4th ed., pp. 43, 60). Rohner Research Publications, Storrs, CT 06268, USA.

[20] Goldberg, D.P. (1972).The detection of psychiatric illness by questionnaire. Oxford University Press, London.

[21] Sherer, M., Maddux, J. E., Mercandante, B., Prentice-Dunn, S., Jacobs, B., \& Rogers, R,W. (1982).The self-efficacy scale: Construction and validation. Psychological Reports. 51, 663-671.

[22] Jasmine, U.H., Uddin, M.K., \& Sultana, S. (2007). Adaptation of Parental Acceptance-Rejection Questionnaire and Personality Assesment Questionnaire in Bangla Language. Bangladeh Psychological Studies, 17, 49-70.

[23] Sarker, N. R., \& Rahman, A. (1989).Occupational stress and mental health of working enviornment, UFG, Dhaka.

[24] Illyas, Q. S. M. (2005). Bangla Version of Shere`s General Self efficacy Scale. Unpublished Manuscript, Department of Psychology, University of Dhaka, Bangladesh.

[25] Khaleque, A., Shirin, A., \& Uddin, M. K. (2011). Attachment relationships and psychological adjustment of married adults. Social Indicators Research, 104, 1-8.

[26] Rohner, R. P., Khaleque, A., Elias, S., \& Sultana, S. (2010). The relationship between perceived teacher and parental acceptance, school conduct, and the psychological adjustment of Bangladeshi adolescents. Cross-Cultural Research, 44(3), $239-252$. doi:10.1177/1069397110366900

[27] Kessler, R.C., Berglund, P., Demler, O., Jin, R., Koretz, D., Merikangas, K.R., Rush, A.J., Walters, E.E., \& Wang, P.S. (2003, June). The epidemiology of major depress sive disorder: results from the National Comorbidity Survey Replication (NCS-R). Journal of the American Medical Association, 289(23), 3095-3105. doi:10.1001/jama.289.23.3095

[28] Miller, R. B., Greene, B. A., Montalvo, G. P., Ravindran, B., \& Nichols, J. D. (1996). Engagement in academic work: The role of learning goals, future consequences, pleasing others, and perceived ability. Contemporary Educational Psychology, 21,388-422.

[29] Khan, S., Hassan, S., Husain,G., \& Iram Gul, I. (2011). Relationship of Parental Acceptance and Rejection with Psychological Wellness in Young Adults. Journal of Rawalpindi Medical College, 15(1), 24-26.

[30] Rohner, R. P., \& Veneziano, R. A. (2001). The importance of father love: History and contemporary evidence. Review of General Psychology, 5, 382-405.

[31] Wilson, K. R., \& Prior, M. R. (2011). Father involvement and child well-being. Journal of Pediatrics and Child Health, 47(7), 405407. doi: $10.1111 / j .1440-1754.2010 .01770 . x$ 